\title{
COULD THE FULLY IMPLANTABLE LIONHEART TM BE A FUTURE SOLUTION CONSIDERING THE LIMITED NUMBER OF DONORS FOR CARDIAC TRANSPLANT?
}

\author{
MARIO VIGANÒ *, ANDREA MARIA D'ARMINI *, \\ CARLO PELLEGRINI *, ALESSIA ALLONI *
}

\begin{abstract}
Cardiac failure constitutes one of the main health problems in western countries. Despite the recent outstanding progress the pharmaceutical treatment of this condition is not suitable to overcome a great percentage of failures and an insufficient quality of life for many patients. The heart transplantation today is considered the most suitable therapeutic option. However, the limited number of donors compared to the great number of patients on the waiting list constitute a major limitation of this solution. The initial experience on the development of cardiac assist devices conceived as a "bridge to the transplantation" based on mechanical circulatory support has provided encouraging results to use such devices (ventricular assist devices) for a long period which could be considered as an acceptable therapeutic solution. The recent technological developments have made available the necessary miniaturization of the various mechanical components of the system for ventricular assistance and the proper solution for energy supply and control. On the light of the positive results obtained the ventricular assist devices can be considered today a permanent solution for a significant number of patients. This chapter illustrates the first Italian experience on the clinical application of the LionHeart, a totally implantable system for ventricular assistance. A concise description of the system and the surgical technique for implantation are provided and the preliminary results on the short term are discussed. Even considering the limited period of observation, this therapeutic option appears to be safe and suitable for a selected population of patients which cannot be considered proper candidates for heart transplantation.
\end{abstract}

*Centro Cardiochirurgico "Charles Dubost”, IRCCS Policlinico "San Matteo" Università degli Studi di Pavia 


\section{INTRODUZIONE}

L'insufficienza cardiaca rappresenta il principale problema riguardante la salute pubblica nel mondo occidentale. La sua incidenza e prevalenza sono in continuo aumento contrariamente a quanto accade per le altre patologie cardiovascolari. Negli Stati Uniti i pazienti affetti da insufficienza cardiaca sono 5.000.000 ed ogni anno si registrano 500.000 nuovi casi [1]. Questa patologia è responsabile della morte di circa 300.000 pazienti ogni anno. In Europa si stima che i pazienti affetti da insufficienza cardiaca siano da 2 a 10 milioni [2]. La mortalità globale a quattro anni è pari a circa il $40 \%$, mentre, ad un anno, la mortalità in pazienti con scompenso cardiaco severo è pari al 50\% [2]. Dal momento che l'insufficienza cardiaca è essenzialmente una patologia dell'età avanzata [3] (approssimativamente dal $6 \mathrm{al} 10 \%$ delle persone di età superiore a 65 anni è affetta da scompenso cardiaco [4]) e che la durata della vita media, nel mondo occidentale, sta aumentando, la prevalenza di questa patologia è destinata ad aumentare nel prossimo futuro.

Malgrado i recenti progressi nella terapia farmacologica dell'insufficienza cardiaca, la sopravvivenza e la qualità della vita dei pazienti rimangono scadenti. Il trapianto di cuore risulta essere un'efficace risorsa terapeutica per l'insufficienza cardiaca in fase avanzata. Sfortunatamente lo scarso numero di donatori riduce la possibilità di offrire quest'opzione terapeutica ad un numero adeguato di pazienti. Ogni anno negli Stati Uniti più di 4.250 pazienti vengono inseriti in lista di attesa per trapianto cardiaco, mentre soltanto 2.500 sono i trapianti effettuati [5]. In Italia nel 2000 il numero di donatori ha soddisfatto solo il 41.5\% delle richieste (706 pazienti in lista di attesa, 293 trapianti effettuati [6]). Globalmente nel mondo occidentale i pazienti iscritti nelle liste d'attesa sono 10.000 ed ogni anno vengono eseguiti circa 4.000 trapianti di cuore, ma almeno 150.000 pazienti sarebbero potenziali candidati al trapianto cardiaco. La grande discrepanza tra "domanda" ed "offerta" ha portato ad un costante aumento del numero di potenziali riceventi con conseguente incremento dei tempi d'attesa (mediana: 2 mesi nel periodo 1985-1988, 14 mesi nel periodo 2000-2002. Dati personali non pubblicati). L'aumento del numero globale di donatori (media nazionale italiana 13,7\% nel 1999 e 14,2\% nel 2000 [6]) non ha portato ad un corrispondente incremento della disponibilità di cuori donati, contrariamente a quanto avvenuto per fegato, rene e pancreas. Infatti i donatori, che prima erano rappresentati prevalentemente da 
giovani deceduti per trauma della strada, attualmente sono soprattutto soggetti di età più avanzata, deceduti per emorragia cerebrale che, pertanto, presentano più frequentemente patologie cardiovascolari in atto. Numerose opzioni terapeutiche sono state via via considerate in alternativa al trapianto di cuore. Tra queste alcune possono essere definite "storiche" (ed alcune sono già state abbandonate) mentre altre sono tuttora in evoluzione. Tra quelle già stabilite ricordiamo la rivascolarizzazione miocardica "estrema", il rimodellamento del ventricolo sinistro, l'anuloplastica valvolare mitralica che, unitamente con l'assistenza meccanica come bridge al trapianto, rivestono ancora un ruolo attuale. Ormai abbandonate, perché gravate nel tempo da un rischio superiore al beneficio, sono la riduzione volumetrica del ventricolo sinistro e la cardiomioplastica dinamica. Quelle in evoluzione ed ancora in fase di studio sono: la terapia cellulare, la terapia genica, lo xenotrapianto e l'assistenza circolatoria meccanica definitiva. Nel nostro Centro, sin dal 1987, le assistenze ventricolari meccaniche sono state utilizzate quale supporto cardiocircolatorio in attesa del trapianto [7, 8]. Durante questo periodo di tempo i "device" sono stati via via modificati al fine di consentire assistenze a lungo termine, mentre gli sviluppi della tecnologia hanno permesso di ridurre notevolmente le dimensioni delle "pompe" e dei "controllori". L'esperienza del "ponte al trapianto" ha dimostrato che le assistenze cardiocircolatorie possono essere realizzate per lunghi periodi di tempo. Il limite a questa applicazione sta nelle complicanze a lungo termine, la maggior parte delle quali dipende dalla presenza di cavi e fili percutanei. I positivi risultati ottenuti dalle esperienze cliniche di device utilizzati come "ponte al trapianto" hanno determinato la nascita del concetto di "supporto permanente" o di "terapia definitiva". Questo studio illustra il successo della prima esperienza clinica italiana di assistenza ventricolare sinistra permanente totalmente impiantabile con il device "LionHeart" ${ }^{\mathrm{TM}}$ ".

\section{MATERIALI E METODI}

\section{POPOLAZIONE}

Dall'Ottobre 2001 al Marzo 2002 quattro pazienti di sesso maschile sono stati sottoposti ad impianto di assistenza ventricolare sinistra tipo 
"LionHeart ${ }^{\mathrm{TM}}$ ", (Arrow International Inc., Reading, Pennsylvania) per insufficienza cardiaca in stadio terminale, come supporto circolatorio permanente. I dati demografici sono illustrati in Tab. 1. L'età media era di $64 \pm 2.5$ anni (intervallo 61-67). Tutti i pazienti sono stati esclusi dalle liste di attesa per trapianto cardiaco a causa dell'età (> 60 anni) e/o di patologie associate (Rettocolite ulcerosa, esofago di Barrett nei pazienti $\mathrm{n}^{\circ} 1 \mathrm{e}^{\circ} 4$ rispettivamente). Tutti i pazienti erano in IV classe NYHA ed il primo paziente, preoperatoriamente era in trattamento con infusione continua di inotropi e vasodilatatori.

Tab. 1.

Dati demografici e pre-operatori dei pazienti a cui è stato impiantato il device LionHeart ${ }^{T M}$ LVAD.

\begin{tabular}{|c|c|c|c|c|c|}
\hline $\begin{array}{c}\text { Paziente } \\
\text { no. }\end{array}$ & Età & Diagnosi & $\begin{array}{c}\text { Chirurgia } \\
\text { Precedente }\end{array}$ & $\begin{array}{c}\text { Preoperatorio } \\
\text { lnotropi/Vasodilatatori }\end{array}$ & $\begin{array}{c}\text { Causa di } \\
\text { esclusione } \\
\text { per H-Tx }\end{array}$ \\
\hline 1 & 64 & CMI & - & $\begin{array}{c}\text { Dobutamina }(5 \mu / \mathrm{kg} / \mathrm{min}) \\
\text { Nitroprussiato di Sodio } \\
(0.6 \mu \mathrm{g} / \mathrm{kg} / \mathrm{min})\end{array}$ & $\begin{array}{c}\text { Età } \\
\text { Rettocolite } \\
\text { ulcerosa }\end{array}$ \\
\hline 2 & 65 & $\mathrm{CMD}$ & - & - & Età \\
\hline 3 & 61 & $\mathrm{CMI}$ & $4 \mathrm{BPAC}$ & - & Età \\
\hline 4 & 67 & $\mathrm{CMI}$ & - & - & $\begin{array}{c}\text { Età } \\
\text { Esofago } \\
\text { di Barrett }\end{array}$ \\
\hline
\end{tabular}

Legenda: $\mathrm{CMI}=$ cardiomiopatia ischemica, $\mathrm{CMD}=$ cardiomiopatia dilatativa, $\mathrm{BPAC}=$ by-pass aortocoronarico.

\section{DESCRIZIONE DEL DEVICE}

\section{Componenti impiantabili}

I componenti impiantabili includono: una pompa pulsatile con cannule di afflusso e di efflusso, un controllore del motore, una camera di compensazione ed una spirale metallica impiantabile che è parte del sistema di trasmissione dell'energia attraverso la cute (Fig. 1). Il "LionHeart ${ }^{\mathrm{TM}}$ ", essendo un sistema totalmente impiantabile, consente di evitare ogni forma di connessione percutanea, eliminando così una costante porta di entrata di agenti patogeni. La pompa, alimentata elettricamente, è costi- 
tuita da un motore collegato ad un meccanismo di piastra "a spinta" imperniata su una vite senza fine, una sacca di poliuretano che viene compressa ritmicamente dalle piastre, espellendo in tal modo il sangue dal ventricolo, due valvole meccaniche a disco, una al termine del condotto di afflusso e l'altra all'inizio del condotto di efflusso, che servono per garantire l'unidirezionalità del flusso. La pompa è collegata all'apice del ventricolo sinistro tramite il condotto di afflusso ed all'aorta ascendente mediante il condotto di efflusso anastomizzato in termino-laterale. Il flusso massimo possibile della pompa è pari a $8 \mathrm{l} / \mathrm{min}$. Il controllore è localizzato in un alloggiamento in titanio con un set di batterie ricaricabili che permettono il distacco dall'alimentazione esterna per approssimativamente 20 minuti. Il controllore, che riceve corrente da un alimentatore esterno, fornisce energia alla pompa, ne governa il funzionamento e consente la rilevazione di dati tramite telemetria.

Il passaggio di energia attraverso la cute avviene per induzione fra la spirale esterna del sistema di traduzione dell'energia e la spirale interna, poste l'una in corrispondenza dell'altra. Il passaggio transcutaneo necessita di una prima trasformazione dell'energia in corrente alternata che successivamente deve essere nuovamente trasformata in corrente continua per alimentare il motore della pompa. La completa impiantabilità del sistema viene garantita dalla comunicazione di quest'ultimo con una camera di compensazione contenente aria. La camera, costituita da una sacca circolare in materiale polimerico e da una porta di infusione dell'aria posta sottocute, compensa lo spostamento di aria prodotto dai movimenti della pompa. È necessario effettuare periodicamente un controllo della pressione del sistema di compensazione mediante la porta di infusione ed eventualmente eseguire il rifornimento di aria. Poiché vi è una diffusione dell'aria attraverso la camera, il sistema necessita di essere rifornito ad intervalli di circa 1 mese.

\section{Componenti esterni}

I componenti esterni includono: un trasmettitore di corrente, la cui funzione principale è di fornire l'energia elettrica attraverso la cute intatta al controllore interno, un sistema di alimentazione portatile, un caricabatterie con relativo pacco batterie, un'antenna telemetrica ed un monitor del sistema. Questi componenti assicurano un funzionamento corretto e continuo della pompa così da permettere ai pazienti di ritornare ad uno stile di vita confortevole dopo la dimissione dall'ospedale. 


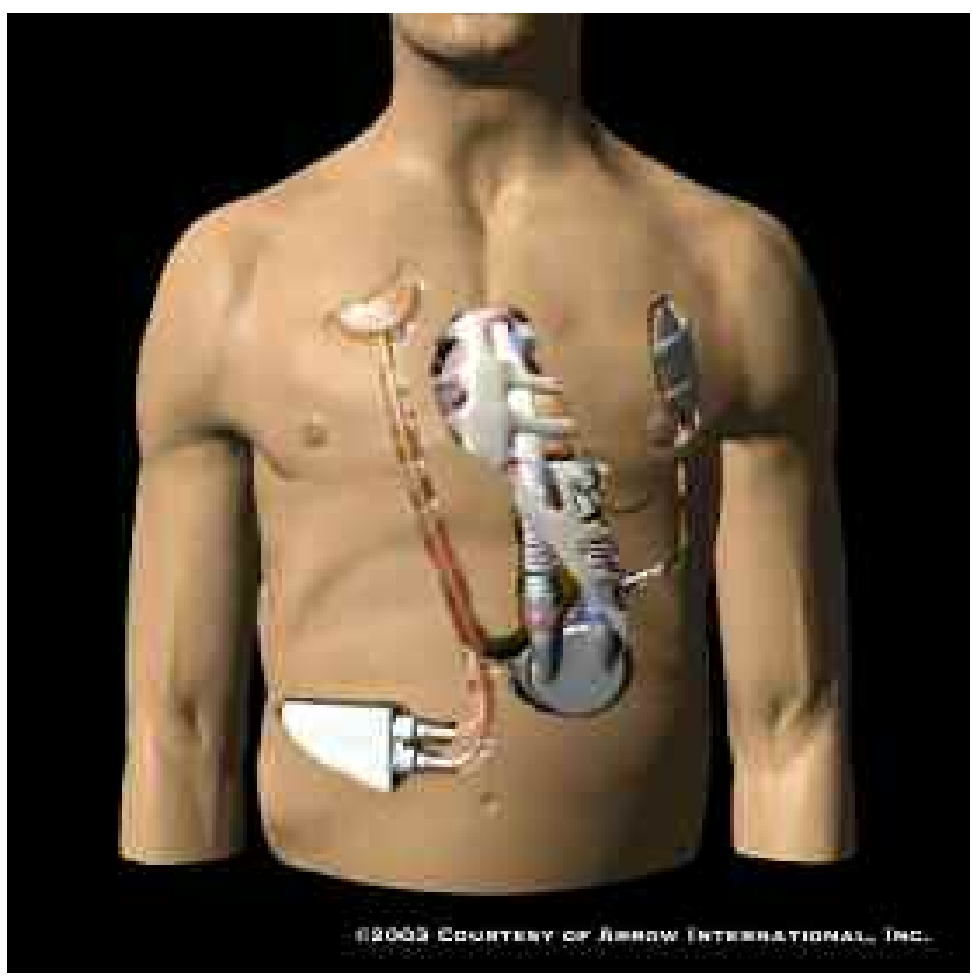

Fig. 1.

Rappresentazione schematica dei componenti del sistema di assistenza ventricolare sinistra Lion Heart e loro rapporti con le strutture toraciche e addominali del paziente.

A partire da sinistra si osservano il controllore della pompa e la spirale interna del sistema di trasmissione transcutanea dell'energia, la pompa con la cannula di efflusso (bullone fucsia) e la cannula di afflusso (bullone blu) ed, infine, la camera di compensazione con il sistema di infusione dell'aria, che viene posizionato in sede sottocutanea sull'emitorace di sinistra. La figura mostra la posizione assunta dai vari componenti interni del sistema al termine dell'intervento chirurgico.

\section{TECNICA DI IMPIANTO}

Si esegue una sternotomia mediana, con un'incisione estesa dal giugulo sino oltre l'ombelico. Viene ricavata una tasca dietro la faccia posteriore dei muscoli della parete addominale nella parte più bassa del quadrante destro, sempre attraverso l'incisione mediana (servirà per l'indovamento del controllore). Una seconda tasca sottocutanea viene confezionata a livello dell'emitorace destro, in sede sottoclaveare, per il posizionamento 
della spirale interna del sistema di trasmissione transcutanea dell'energia. La tasca per l'alloggiamento della pompa è ricavata sempre attraverso l'incisione mediana, a sinistra della linea alba, in zona paramediana, dietro la fascia posteriore dei muscoli retti. La dissezione viene proseguita lateralmente verso sinistra al fine di ottenere un alloggiamento adatto alla pompa tra la fascia posteriore dei muscoli retti ed il peritoneo.

Si inizia la circolazione extra-corporea mantenendo il paziente in normotermia per tutta la durata dell'intervento. Il sito per la cannulazione apicale è normalmente localizzato appena anteriormente all'apice cardiaco. In tale sede vengono passati circonferenzialmente 10-12 punti staccati ad $\mathrm{U}$ su feltro di teflon con fili non riassorbibili in Ethibond 0 così da creare, intorno all'apice, una circonferenza di circa $3-4 \mathrm{~cm}$ di diametro. Dopo aver creato un'apertura al centro della circonferenza delineata attorno all'apice, il manicotto apicale viene assicurato in posizione mediante i punti di ancoraggio precedentemente passati. La cannula di afflusso viene in seguito posizionata attraverso un'incisione di circa $3 \mathrm{~cm}$ condotta sul diaframma in modo che la sua estremità distale venga a trovarsi in corrispondenza del manicotto. La pompa assemblata viene posizionata nell'apposita tasca e la cannula di afflusso viene collegata con il manicotto fissato all'apice del ventricolo sinistro. Si può quindi procedere alla deareazione della pompa. Nel frattempo vengono prese le misure per la cannula di efflusso che viene, poi, sezionata. Dopo il posizionamento di un clampo tangenziale sull'aorta, è possibile eseguire un'anastomosi termino-laterale tra la cannula e la parete del vaso mediante sutura continua con polipropilene $4 / 0$. Il graft viene deareato tramite la rimozione del clampo aortico. La deareazione si completa utilizzando un ago da 18-gauge posizionato nella cannula di efflusso, quindi viene rimosso il clampo dalla protesi di efflusso. Si procede, poi, alla connessione del controllore con la pompa e la spirale interna. I cavi elettrici che connettono la pompa al controllore vengono tunnellizzati sino alla tasca ove questo è alloggiato. La spirale esterna è fissata con due punti singoli alla cute. Si attiva, quindi, la pompa e si inizia lo svezzamento del paziente dalla circolazione extra-corporea aumentando via via il supporto della pompa.

Quando il funzionamento della pompa è stabile, si posiziona la camera di compensazione nello spazio pleurico sinistro, fra il polmone e la fascia endotoracica, dopo ampia apertura della pleura corrispondente. La connessione viene collegata alla pompa. La porta di immissione dell'aria viene posta in un alloggiamento sottocutaneo della parete toracica sinistra e le connessioni vengono tunnellizzate sino alla camera di compensazione attraverso il corrispondente spazio intercostale. Infine, si po- 
sizionano i drenaggi (uno per la tasca della pompa, uno per la tasca del controllore, due per il mediastino) e l'incisione viene chiusa per strati separati. Il paziente è quindi trasferito in terapia intensiva dove si procede ad un ulteriore controllo della camera di compensazione.

\section{PROTOCOLLO PER LA TERAPIA ANTICOAGULANTE}

Per la presenza di valvole meccaniche e l'impiego di materiale protesico è necessario che i pazienti a cui sia stata impiantata un'assistenza ventricolare meccanica tipo LionHeart ${ }^{\mathrm{TM}}$ assumano una terapia anticoagulante. Nelle prime fasi questa è rappresentata dall'infusione continua di eparina, iniziata allorché il sanguinamento post-operatorio si riduce. La posologia viene modificata in modo da mantenere valori di tempo parziale di tromboplastina da 1.5 a 2 volte superiori al valore normale. Non appena la ventilazione meccanica non è più necessaria e la peristalsi intestinale è ripresa, si somministra warfarin al fine di ottenere valori di INR compresi fra 2.5 e 4.0. Benché l'uso di farmaci inibenti l'aggregazione piastrinica non sia considerato obbligatorio, il paziente assume abitualmente ticlopidina al fine di prevenire fenomeni di micro-aggregazione.

\section{RISULTATI}

\section{DECORSO POST-OPERATORIO}

Al momento attuale (Maggio 2002), non vi sono stati casi di mortalità operatoria né ospedaliera. In tutti i casi si è reso necessario un periodo prolungato di supporto inotropico con dobutamina (media: $28 \pm 17.8$ giorni). Inoltre, in tutti i pazienti si è dovuto infondere nitroprussiato e/o somministrare ossido nitrico (paziente $\mathrm{n}^{\circ} 1$ ) allo scopo di abbattere le resistenze vascolari polmonari e, quindi, favorire la funzione del ventricolo destro. La durata media della ventilazione meccanica è stata di $12 \pm 16$ giorni. Il paziente $\mathrm{n}^{\circ} 3$ è stato sottoposto a tracheostomia a causa del prolungato supporto ventilatorio, mentre per il paziente $\mathrm{n}^{\circ} 4$ si è reso necessario un nuovo periodo di ventilazione meccanica a partire dalla VII giornata post-operatoria a causa dell'insorgenza di insufficienza respiratoria acuta. La degenza media in terapia intensiva è stata 
di $16.8 \pm 13.6$ giorni. Due pazienti $\left(n^{\circ} 1\right.$ e $\left.n^{\circ} 2\right)$ sono stati dimessi dall'ospedale dopo una riabilitazione fisica ed un training sul corretto impiego dei componenti del sistema. Ad oggi la durata media dell'assistenza circolatoria è di 79 giorni (intervallo: 17-157).

\section{EMODINAMICA}

I dati emodinamici sono riportati nella Tab. 2. Nella Tab. 3 sono, inoltre, schematizzati i confronti tra i principali parametri prima e dopo l'impianto del device. L'efficacia di questa assistenza nel ripristinare le normali condizioni cardiocircolatorie è chiaramente evidente.

Tab. 2.

Dati emodinamici pre- e post-impianto.

\begin{tabular}{|c|c|c|c|c|c|c|c|c|c|c|c|}
\hline & \multicolumn{9}{|c|}{ PRE-IMPIANTO } & \multicolumn{3}{|c|}{ POST-IMPIANTO (giorno 1) } & $\begin{array}{c}\text { POST- } \\
\text { IMPIANT0 } \\
\text { (giorno 14) }\end{array}$ & $\begin{array}{c}\text { POST- } \\
\text { IMPIANT0 } \\
\text { (giorn 30) }\end{array}$ & $\begin{array}{c}\text { POST- } \\
\text { IMPIANT0 } \\
\text { (giorno 90) }\end{array}$ \\
\hline $\begin{array}{c}\text { Pz } \\
\text { no. }\end{array}$ & C.I. & $\begin{array}{c}\text { PAP } \\
\text { (media) }\end{array}$ & PCWP & $\begin{array}{c}\text { PVR } \\
\text { (WU) }\end{array}$ & C.I. & $\begin{array}{c}\text { PAP } \\
\text { (media) }\end{array}$ & PCWP & $\begin{array}{c}\text { PVR } \\
\text { (WU) }\end{array}$ & C.I. & C.I. & C.I. \\
\hline $\mathbf{1}$ & 1,0 & 36 & 27 & 5,6 & 2,3 & 23 & 12 & 3,1 & 2,5 & 2,2 & 2,5 \\
\hline $\mathbf{2}$ & 1,2 & 34 & 27 & 3,1 & 2,4 & 22 & 8 & 3,3 & 3,2 & 3,1 & 2,8 \\
\hline $\mathbf{3}$ & 1,0 & 31 & 25 & 3,3 & 2,8 & 20 & 10 & 2,0 & 3,1 & 2,6 & \\
\hline $\mathbf{4}$ & 1,8 & 20 & 9 & 3,1 & 2,2 & 21 & 10 & 2,6 & 2,5 & & \\
\hline
\end{tabular}

Legenda: $\mathrm{Pz}=$ paziente, C.I. $=$ indice cardiaco, $\mathrm{PAP}=$ pressione arteriosa polmonare espressa in $\mathrm{mmHg}, \mathrm{PCWP}=$ pressione di incuneamento polmonare espressa in $\mathrm{mmHg}, \mathrm{PVR}=$ resistenze vascolari polmonari espresse in unità Wood.

Tab. 3.

Confronto tra i principali valori emodinamici prima e dopo l'impianto del device LionHeart ${ }^{T M}$. A causa del piccolo numero di casi non è stato possibile effettuare analisi statistiche.

\begin{tabular}{|l|c|c|}
\hline & PRE IMPIANTO & POST IMPIANTO \\
\hline C.I. & $1.2 \pm 0.4$ & $2.6 \pm 0.3$ \\
\hline PAP (media) & $30 \pm 7$ & $21 \pm 1$ \\
\hline PCWP & $22 \pm 9$ & $10 \pm 2$ \\
\hline PVR (WU) & $3.8 \pm 1.3$ & $2.7 \pm 0.6$ \\
\hline
\end{tabular}

Legenda: vedi Tab. 2. 


\section{COMPLICANZE}

Paziente $n^{\circ} 1$

Si sono verificati due episodi tromboembolici. Durante la XIV giornata postoperatoria il paziente ha mostrato un deficit del linguaggio ed una paresi del braccio destro causati da un attacco ischemico transitorio. $\mathrm{La}$ tomografia computerizzata ha, infatti, mostrato una lesione a livello della capsula interna sinistra. L'episodio si è risolto 12 ore dopo la ripresa della somministrazione di eparina per via endovenosa. Nel corso della XXVII giornata post-operatoria si è verificato un nuovo attacco ischemico transitorio risoltosi spontaneamente dopo circa 6 ore. In questo caso non è stata eseguita alcuna indagine tomografica di conferma.

Inoltre, in XV giornata post-operatoria, il paziente è stato sottoposto, in anestesia locale, a sutura dell'estremo distale della ferita cutanea a seguito di deiscenza circoscritta della cute.

A circa due mesi dall'impianto dell'assistenza ventricolare, a seguito della riattivazione della colite ulcerosa, si è verificato un episodio di sanguinamento intestinale profuso. Verosimilmente gli elevati valori di anticoagulazione del paziente $(\mathrm{INR}=6.7)$ hanno giocato un ruolo preponderante nella genesi di complicazione. A causa dell'emorragia si è reso necessario trasfondere il paziente con 6 unità di globuli rossi e 4 unità di plasma fresco congelato. Sono stati inoltre somministrati steroidi e salazopirina per la patologia di base. Il sanguinamento si è prontamente ridotto ed è cessato alcuni giorni dopo.

\section{Paziente $n^{\circ} 2$}

Nel corso della I giornata post-operatoria il paziente è stato trattato con defibrillazione cardiaca a causa di un episodio di fibrillazione ventricolare. In X giornata il paziente ha mostrato deficit del linguaggio, la cui causa è stata riconosciuta in un attacco ischemico transitorio risoltosi spontaneamente dopo circa 6 ore.

\section{Pazienti $n^{\circ} 3$ e $n^{\circ} 4$}

Entrambi i pazienti hanno avuto problemi di esaurimento muscolare e conseguente insufficienza respiratoria dopo la cessazione della ventilazione meccanica. A causa degli insoddisfacenti scambi respiratori, in XXI giornata post-operatoria, il paziente $n^{\circ} 3$ è stato tracheostomizzato. Questo paziente necessita ancora del supporto ventilatorio mec- 
canico. Il paziente $n^{\circ} 4$ è stato definitivamente autonomizzato dalla ventilazione meccanica 7 giorni dopo.

\section{DiSCUSSIONE}

In questo studio abbiamo voluto presentare la nostra iniziale esperienza con il device totalmente impiantabile tipo LionHeart ${ }^{\mathrm{TM}}$. Tutti i pazienti sono vivi e due dei quattro (pazienti $n^{\circ} 1$ e 2 ) sono stati già dimessi dall'ospedale ed hanno ripreso le normali attività della vita quotidiana. $\mathrm{Ne}-$ gli anni recenti gli sviluppi tecnologici hanno reso le assistenze ventricolari sicure ed efficaci nello svolgere la loro funzione di "ponte al trapianto". Pertanto, dal momento che il supporto circolatorio meccanico aiuta anche a normalizzare la funzionalità epatica e renale, assicurando al paziente migliori possibilità di superare lo stress chirurgico del trapianto, è iniziato un ampio utilizzo delle assistenze ventricolari meccaniche in tutto il mondo [9]. Sfortunatamente l'assistenza meccanica, anche se di lunga durata, non può modificare il numero totale di trapianti, che dipende esclusivamente dal numero di donatori. L'uso di un sistema di supporto cardiocircolatorio permanente, totalmente impiantabile, potrebbe invece far fronte alla scarsità di donatori, che risulta essere l'unica limitazione al trattamento chirurgico sostitutivo delle insufficienze cardiache in stadio terminale. Inoltre, un sistema come il device LionHeart ${ }^{\mathrm{TM}}$ potrebbe assicurare un trattamento a tutti quei pazienti che non possono essere inseriti in lista d'attesa per trapianto a causa dell'età o di comorbidità.

Uno dei limiti al largo impiego delle assistenze ventricolari meccaniche è rappresentato dai fenomeni tromboembolici [10]. In questo studio, benché si siano verificati problemi di lieve entità ed il tempo di osservazione sia stato breve, abbiamo dimostrato che l'applicazione di un semplice protocollo di anticoagulazione può rendere l'assistenza meccanica più sicura. È noto, infatti, che in corso di supporto circolatorio meccanico, l' $80 \%$ degli episodi tromboembolici avviene nei primi 60 giorni dopo l'impianto [11].

La totale impiantabilità del sistema LionHeart ${ }^{\mathrm{TM}}$ riconosce due vantaggi: 1. l'assenza di cavi percutanei riduce marcatamente l'insorgenza di infezioni, abbassando di molto la morbilità tipicamente associata agli altri tipi di assistenze ventricolari meccaniche; 2. l'assenza di reazioni immunologiche consente di evitare qualunque tipo di immu- 
nosoppressione. È verosimile che questo si ripercuota positivamente sull'insorgenza di tumori, complicazione frequente dei pazienti trapiantati, soprattutto se anziani, dal momento che l'età al momento del trapianto costituisce il principale fattore di rischio per la patologia neoplastica [12].

\section{BIBLIOGRAFIA}

[1] O' Connel JB, Bristow M. "Economic impact of heart failure in the United States: time for a different approach”. J Heart Lung Transplant 1994; 13: S107-12.

[2] Guidelines for the diagnosis of heart failure. The task force on heart failure of the European Society of Cardiology. Eur Heart J 1995; 16: 741-51.

[3] Kannel WB, Belanger AJ. "Epidemiology of heart failure". Am Heart J 1991; 121: 951-7.

[4] Kannel WB. "Epidemiology and prevention of cardiac failure: Framingham Study insights". Eur Heart J 1987; 8 Suppl F: 23-6.

[5] United Network for Organ Sharing. "1997 annual report of the US Scientific Registry of Transplant Recipients and the Organ Procurement and Transplantation Network". Washington, DC: Department of Health and Human Services, 1998.

[6] Centro Nazionale Trapianti. "Attività di donazione, prelievo e trapianto di organi in Italia. Anni 1992-2000. Le cifre". Roma, 2001.

[7] Viganò M, Martinelli L, Minzioni G, Rinaldi M, Pagani F. "Modified method for Novacor left ventricular assist device implantation" Ann Thorac Surg 1996; 61: 247-9.

[8] Di Bella I, Pagani F, Banfi C, Ardemagni E, Capo A, Klersy C, Viganò M. "Results with the Novacor assist system and evaluation of long-term assistance" Eur J Cardiothorac Surg 2000; 18: 112-6.

[9] Frazier OH, Macris MP, Myers TJ, Duncan JM, Radovancevic B, Parnis SM, Cooley DA. "Improved survival after extended bridge to cardiac transplantation". Ann Thorac Surg 1994; 57: 1416-22.

[10] Moskowitz AJ, Weinberg AD, Oz MC, Williams DL. "Quality of life with an implanted left ventricular assist device. Ann Thorac Surg 1997; 64: $1764-9$. 
[11] Smith AJ, Oyer PE. "Development of the Novacor left ventricular assist device". In Shumway SJ, Shumway NE, eds: Thoracic transplantation. Boston, Blackwell Science, inc. 1995: 134-40.

[12] Rinaldi M, Pellegrini C, D’Armini AM, Aiello M, Negri M, Arbustini E, Ippoliti G, Vigano M. "Neoplastic disease after heart transplantation: single centre experience" Eur J Cardiothorac Surg 2001; 19: 696-701.

[13] Pagani F, Ardemagni E, Gazzoli F, Viganò M. "Artificial heart: the moment and the future" Artif. Cells Blood Substit Immobil Biotechnol. 2003. 
